\title{
PARTIAL CROSSED PRODUCTS AS EQUIVALENCE RELATION ALGEBRAS
}

\author{
VIVIANE M. BEUTER AND DANIEL GONÇALVES
}

\begin{abstract}
For a free partial action of a group in a set we realize the associated partial skew group ring as an algebra of functions with finite support over an equivalence relation and we use this result to characterize the ideals in the partial skew group ring. This generalizes, to the purely algebraic setting, the known characterization of partial $C^{*}$ crossed products as groupoid $C^{*}$-algebras. For completeness we include a new proof of the $C^{*}$ result for free partial actions.
\end{abstract}

1. Introduction. The groupoid approach to $C^{*}$-algebras given by Renault in [18] is one of the main concepts in the modern theory of operator algebras, as an ever growing number of $C^{*}$-algebras may be realized and studied as groupoid $C^{*}$-algebras (AF algebras, Cuntz and Cuntz-Krieger algebras, tilings $C^{*}$-algebras are just a few we can mention, see $[\mathbf{1}, \mathbf{8}, \mathbf{9}]$ ). Fourteen years after the work of Renault, the notion of partial actions was introduced by Exel (see [4]) and McClanahan (see [14]), and, as many $C^{*}$-algebras were expressed and studied as partial crossed products (AF algebras, Cuntz-Krieger algebras of infinite matrices. Bunce-Deddens algebras are one of a few examples we can mention (see [1]). Due to these researchers, the theory grew in importance. In 2004, Abadie (see [1]) established that every partial crossed product of a commutative $C^{*}$-algebra can be seen as a groupoid $C^{*}$-algebra. In 2005, Dokuchaev and Exel (see [3]) started the study of purely algebraic partial actions and their associated partial skew group rings, providing a new insight into the theory of partial actions, which was followed by a broadening of the knowledge in the field, see $[\mathbf{2}, \mathbf{3}, \mathbf{5}, \mathbf{7}, \mathbf{1 2}, \mathbf{1 9}]$, for example.

2010 AMS Mathematics subject classification. Primary 16S35, 46L65.

Keywords and phrases. Partial crossed products, equivalence relation, algebras, $C^{*}$-algebras.

The second author was partially supported by CNPq-Brazil.

Received by the editors on December 31, 2012, and in revised form on May 20, 2014. 
It is interesting to note that many results in the theory of $C^{*}$ partial crossed products have equivalent versions in the purely algebraic setting and, as the theory develops, the interaction between the areas increases. Although the study of partial skew group rings is still underdeveloped when compared with its $C^{*}$-counterpart, we can mention a few examples where the interaction between the areas seems to be beneficial to both. For example, Proposition 2.1 of [6] (where partial actions of countable groups are characterized over second countable, compact spaces whose envelope space is Hausdorff) can be obtained, see [6, Remark 2.2], from the algebraic version described in [3]. Also, conditions for simplicity of skew group rings and applications to topological dynamics (and hence to the associated $C^{*}$-algebras) have been studied in $[\mathbf{1 5}, \mathbf{1 6}]$. Some of these results have recently been generalized to partial skew group rings, with applications to partial actions on compact sets (see $[\mathbf{1 0}, \mathbf{1 3}])$.

In this paper, we intend to give purely algebraic versions of some known results in the theory of $C^{*}$ - partial crossed products. In particular, to give the reader some motivation, we start with a simpler, and algebraic in flavor, proof of Abadie's characterization of $C^{*}$-partial crossed products as groupoid algebras, for the case of free partial actions of countable groups acting on commutative $C^{*}$-algebras. In this case the groupoid can be seen as an étale equivalence relation and, as a consequence, the construction of the groupoid $C^{*}$-algebra falls within reach of a much bigger audience. In the second part of the paper we generalize the well-known relation between partial dynamical systems and $C^{*}$-partial dynamical systems to the purely algebraic level. We then proceed to generalize, to the algebraic level, Abadie's result, that is, we show that the partial skew group ring associated to a free algebraic partial action on a set is isomorphic to an algebra of functions with finite support over an equivalence relation. We finish the paper showing how to use the characterization just mentioned to obtain a oneto-one correspondence between ideals and $R$-invariant subsets. In light of recent results characterizing Leavitt path algebras as partial skew group rings, see [12], it is interesting to note that one can use this last correspondence to derive the known ideal structure (see [20]) of Leavitt path algebras associated to finite graphs with no cycles. Before we proceed, we recall, for the reader's convenience, some key definitions below. 
Definition 1.1. A partial action of a group $G$ on a set $\Omega$ is a pair $\theta=\left(\left\{\Delta_{t}\right\}_{t \in \mathrm{G}},\left\{h_{t}\right\}_{t \in \mathrm{G}}\right)$, where, for each $t \in \mathrm{G}, \Delta_{t}$ is a subset of $\Omega$ and $h_{t}: \Delta_{t^{-1}} \rightarrow \Delta_{t}$ is a bijection such that:

(1) $\Delta_{e}=\Omega$ and $h_{e}$ is the identity in $\Omega$;

(2) $h_{t}\left(\Delta_{t^{-1}} \cap \Delta_{s}\right)=\Delta_{t} \cap \Delta_{t s}$;

(3) $h_{t}\left(h_{s}(x)\right)=h_{t s}(x), x \in \Delta_{s^{-1}} \cap \Delta_{s^{-1} t^{-1}}$.

If $\Omega$ is a topological space, we also require that each $\Delta_{t}$ be an open subset of $\Omega$ and that each $h_{t}$ be a homeomorphism of $\Delta_{t^{-1}}$ onto $\Delta_{t}$.

Analogously, a pair $\theta=\left(\left\{D_{t}\right\}_{t \in \mathrm{G}},\left\{h_{t}\right\}_{t \in \mathrm{G}}\right)$ is a partial action of $\mathrm{G}$ on an algebra $\mathrm{A}$ if each $D_{t}$ is a closed two-sided ideal and each $h_{t}$ is an isomorphism of $D_{t^{-1}}$ onto $D_{t}$. In the case where $\mathrm{A}$ is a $C^{*}$-algebra we also require that each $h_{t}$ be a ${ }^{*}$-isomorphism.

Definition 1.2. A partial action $\left(\left\{\Delta_{t}\right\}_{t \in \mathrm{G}},\left\{h_{t}\right\}_{t \in \mathrm{G}}\right)$ is said to be free if, for all $x \in \Omega, h_{t}(x)=x$ implies that $t=e$, where $e$ is the group unit.

It is well known that the category of partial actions on a Hausdorff locally compact space $\mathrm{X}$ is equivalent to the category of partial actions on the $C^{*}$-algebra of the continuous functions vanishing at infinity $\mathrm{C}_{0}(\mathrm{X})$ (see [1, Proposition 1.5], for example). In our work, we will use that, given a partial action $\left(\left\{\mathrm{X}_{t}\right\}_{t \in G},\left\{h_{t}\right\}_{t \in G}\right)$ of $\mathrm{G}$ on $\mathrm{X}$, then $\left(\left\{\mathrm{C}_{0}\left(\mathrm{X}_{t}\right)\right\}_{t \in G},\left\{\alpha_{t}\right\}_{t \in G}\right)$, where $\alpha_{t}: \mathrm{C}_{0}\left(\mathrm{X}_{t^{-1}}\right) \rightarrow \mathrm{C}_{0}\left(\mathrm{X}_{t}\right)$, given by $\alpha_{t}(f)=f \circ h_{t}^{-1}$, is a partial action of $\mathrm{G}$ on $\mathrm{C}_{0}(\mathrm{X})$. Next we recall the definition of the partial crossed product.

Definition 1.3. Let $\theta=\left(\left\{D_{t}\right\}_{t \in \mathrm{G}},\left\{\alpha_{t}\right\}_{t \in \mathrm{G}}\right)$ be a partial action of $\mathrm{G}$ on the $C^{*}$-algebra $\mathrm{A}$. Then the partial crossed product of A by $\mathrm{G}$, denoted by $A \rtimes_{\alpha} G$, is the enveloping $C^{*}$-algebra of $\mathcal{L}$, where $\mathcal{L}$ is the

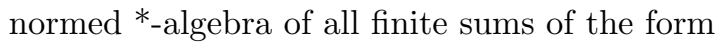

$$
\mathcal{L}=\left\{\sum_{t \in G} a_{t} \delta_{t}: a_{t} \in \mathcal{D}_{t}\right\} \subseteq l_{1}(G, A),
$$

where, for $a=\left(a_{t}\right)_{t \in G} \in \mathcal{L}$ and $b=\left(b_{t}\right)_{t \in G} \in \mathcal{L}$, multiplication, involution and norm are defined by $(a * b)_{\gamma}=\sum_{t \in G} \alpha_{t}\left(\alpha_{t^{-1}}\left(a_{t}\right) b_{t^{-1} \gamma}\right)$, $\left(a^{*}\right)_{\gamma}=\alpha_{\gamma}\left(a_{\gamma^{-1}}^{*}\right)$ and $\|a\|=\sum_{t \in G}\left\|a_{t}\right\|$. 
Another key definition we need to recall is that of an étale equivalence relation. In the language of [18] this is an $r$-discrete groupoid with counting measure as a Haar system. In our context, an étale equivalence relation $R \subseteq \mathrm{X} \times \mathrm{X}$, where $\mathrm{X}$ is locally compact Hausdorff, is one that can be equipped with two maps, called range and source, defined by $r(x, y)=x$ and $s(x, y)=y$ such that $R$ is $\sigma$-compact, $\Delta=\{(x, x) \in R: x \in \mathrm{X}\}$ is an open subset of $R$ and, for all $(x, y) \in R$, there exists a neighborhood $\mathrm{U}$ of $(x, y)$ in $R$ such that $r$ restricted to $\mathrm{U}$ and $s$ restricted to $\mathrm{U}$ are homeomorphisms from $\mathrm{U}$ onto open subsets of $\mathrm{X}$, see also [17].

Finally, given an étale equivalence relation $R$ over a locally compact set $\mathrm{X}$, the groupoid algebra associated to it is obtained as the completion, over a certain norm (see $[\mathbf{8}, \mathbf{1 8}]$ ), of the *-algebra of the continuous functions with compact support in $R, \mathrm{C}_{c}(R)$, where the *-algebra operations are defined, for $f, g \in \mathrm{C}_{c}(R)$, as

$$
f * g(x, z)=\sum_{y \in[x]} f(x, y) g(y, z)
$$

and

$$
f^{*}(x, y)=\overline{f(y, x)}
$$

where $[x]$ denotes the equivalence class of $x$.

2. $C^{*}$-algebra level. Let $\left(\left\{X_{t}\right\}_{t \in \mathrm{G}},\left\{h_{t \in \mathrm{G}}\right\}\right)$ be a free partial action of a countable group $\mathrm{G}$ on a locally compact Hausdorff space $\mathrm{X}$ such that $X_{t}$ is $\sigma$-compact for every $t$. In this section, we prove that the partial crossed product $\mathrm{C}_{0}(X) \rtimes_{\alpha} G$ associated to the corresponding partial action $\left(\left\{\mathrm{C}_{0}\left(\mathrm{X}_{t}\right)\right\}_{t \in G},\left\{\alpha_{t}\right\}_{t \in G}\right)$ (as defined in the introduction) is isomorphic to the full groupoid $C^{*}$-algebra $\mathrm{C}^{*}(R)$, where $R$ is defined below.

Definition 2.1. We say that $x$ is equivalent to $y, x \sim y$, if there exists $t \in G$ such that $x \in X_{t^{-1}}$ and $h_{t}(x)=y$.

Remark 2.2. Notice that the elements of $R$ are of the form $\left(x, h_{t}(x)\right)$ and, since the action is free, for each $(x, y) \in R$, there exists one and only one $t \in G$ such that $(x, y)=\left(x, h_{t}(x)\right)$. 
Of course, we are still missing the key ingredient before we can proceed. That is the topology of $R$. We stress that this is not the topology inherited from the product topology of $\mathrm{X} \times \mathrm{X}$. Instead, to obtain an étale equivalence relation, we give $R$ the inherited topology from $\mathrm{X} \times \mathrm{G}$ (with the product topology) via the map $\left(x, h_{t}(x)\right) \in R \mapsto$ $(x, t) \in \mathrm{X} \times \mathrm{G}$. Notice that, since the action is free, this map is injective. So, a sequence $\left\{\left(x_{n}, h_{t_{n}}(x)\right\}\right.$ converges to $\left(x, h_{t}(x)\right) \in R$ if and only if $\left\{x_{n}\right\}$ converges to $x$ in $\mathrm{X}$ and $t_{n}$ are eventually all equal to $t$. With this topology, we can now prove that $R$ is étale.

Proposition 2.3. $R$ is an étale equivalence relation.

Proof. Before we show that $R$ is étale we should prove that $R$ is an equivalence relation. It is straightforward to check that $R$ is symmetric and reflexive. We show that $R$ is transitive below.

If $x \sim y$ and $y \sim z$, then there exist $t, s \in G$ such that $x \in X_{t^{-1}}$, $y \in X_{t} \cap X_{s^{-1}}, z \in X_{s}, h_{t}(x)=y$ and $h_{s}(y)=z$. This implies that

$$
\begin{aligned}
& x \in h_{t}^{-1}\left(X_{t} \cap X_{s^{-1}}\right) \subseteq X_{(s t)^{-1}}, \\
& z \in h_{s}\left(X_{s^{-1}} \cap X_{t}\right)=X_{s} \cap X_{s t}
\end{aligned}
$$

and

$$
z=h_{s}(y)=h_{s}\left(h_{t}(x)\right)=h_{s t}(x) .
$$

Taking $r=s t$, we have that $x \in X_{r^{-1}}, z \in X_{r}$ and $h_{r}(x)=z$. We conclude that $x \sim z$.

Now we prove that $R$ is étale. By hypothesis, we have that $X_{t}$ is $\sigma$-compact for all $t \in G$. Then, for each $t$, there exists a countable family of compact subsets of $X_{t},\left\{K_{n}^{t}\right\}_{n \in \mathbb{N}}$, such that

$$
X_{t}=\bigcup_{n \in \mathbb{N}} K_{n}^{t}
$$

We conclude that the sets

$$
U_{t, n}:=\left\{\left(x, h_{t}(x)\right): x \in K_{n}^{t}\right\}
$$

are compact and

$$
R=\bigcup_{\substack{t \in G \\ n \in \mathbb{N}}} U_{t, n}
$$


is $\sigma$-compact. That the diagonal $\triangle=\left\{\left(x, h_{e}(x)\right): x \in X\right\}=U_{e}$ is open in $R$ is clear. Finally, if $\left(x, h_{t}(x)\right) \in R$, then $U_{t}:=\left\{\left(z, h_{t}(z)\right)\right.$ : $\left.z \in X_{t^{-1}}\right\}$ is an open neighborhood of $\left(x, h_{t}(x)\right)$ and the range $r: U_{t} \rightarrow$ $X_{t^{-1}}$ and source $s: U_{t} \rightarrow X_{t}$ maps are homeomorphisms.

Remark 2.4. Notice that, since the partial action $\left(\left\{X_{t}\right\}_{t \in \mathrm{G}},\left\{h_{t \in \mathrm{G}}\right\}\right)$ is free, the map $(x, y) \mapsto(y, t, x)$, where $t$ is the unique element of $\mathrm{G}$ such that $h_{t}(x)=y$, is an isomorphism from $R$ onto $\mathcal{G}$, where $\mathcal{G}$ is the groupoid constructed in [1].

We can now consider $\mathrm{C}^{*}(R)$ and show that it is isomorphic to $\mathrm{C}_{0}(X) \rtimes_{\alpha} G$. Our proof relies on the theory of core sub-algebras, so that, by [6, Proposition 3.4], it is enough to show that $\mathcal{L}$ and $\mathrm{C}_{c}(R)$ are dense core sub-algebras of $\mathrm{C}_{0}(X) \rtimes_{\alpha} G$ and $\mathrm{C}^{*}(R)$, respectively, and that they are isomorphic *-algebras. For the reader's convenience, we recall the definition of a core sub-algebra below.

Definition 2.5. Let $A$ be a $C^{*}$-algebra, and let $B \subseteq A$ be a (not necessarily closed) ${ }^{*}$-subalgebra. We say that $B$ is a core subalgebra of $A$ when every representation ${ }^{1}$ of $B$ is continuous relative to the norm induced from $A$.

As a consequence of our next result we obtain that $\mathcal{L}$ is a core subalgebra of $\mathrm{C}_{0}(X) \rtimes_{\alpha} G$. For the analogous result concerning $\mathrm{C}_{c}(R)$ we refer the reader to $[\mathbf{6}, \mathbf{1 8}]$.

Proposition 2.6. Let $\left(\left\{D_{t}\right\}_{t \in G},\left\{\alpha_{t \in G}\right\}\right)$ be a partial action of a discrete group $\mathrm{G}$ over the $C^{*}$-algebra $A$. Then $\mathcal{L}$ is a core sub-algebra of $A \rtimes_{\alpha} G$.

Proof. Let $\lambda$ be a representation of $(\mathcal{L},|\|\cdot\||)$. We have to show that $\|\lambda(a)\| \leq|\|a\||$ for all $a \in \mathcal{L}$, where

$$
|\|a\||=\sup _{\pi}\left\{\|\pi(a)\|: \pi \text { is a representation such that }\|\pi(a)\| \leq\|a\|_{l_{1}}\right\} .
$$

Notice that $A_{0}=\left\{a \delta_{0} \in \mathcal{L}: a \in A\right\}$, equipped with the $\mathcal{L}$ operations, is a $C^{*}$-algebra (isomorphic to $\mathrm{A}$ ) and so $\left\|\lambda\left(a \delta_{0}\right)\right\| \leq\left\|a \delta_{0}\right\|_{l_{1}}$ for all 
$a \delta_{0} \in \mathcal{L}$. It follows that

$$
\begin{aligned}
\left\|\lambda\left(a_{t} \delta_{t}\right)\right\|^{2} & =\left\|\lambda\left(a_{t} \delta_{t}\right) \lambda^{*}\left(a_{t} \delta_{t}\right)\right\|=\left\|\lambda\left(\left(a_{t} \delta_{t}\right)\left(a_{t} \delta_{t}\right)^{*}\right)\right\| \\
& =\left\|\lambda\left(\left(a_{t} \delta_{t}\right) \alpha_{t^{-1}}\left(a_{t}^{*}\right) \delta_{t^{-1}}\right)\right\|=\left\|\lambda\left(a_{t} a_{t}^{*} \delta_{0}\right)\right\| \\
& \leq\left\|a_{t} a_{t}^{*} \delta_{0}\right\|=\left\|a_{t} a_{t}^{*}\right\|=\left\|a_{t}\right\|^{2}=\left\|a_{t} \delta_{t}\right\|^{2}
\end{aligned}
$$

and hence $\left\|\lambda\left(a_{t} \delta_{t}\right)\right\| \leq\left\|a_{t} \delta_{t}\right\|_{l_{1}}$ for all $a_{t} \delta_{t} \in \mathcal{L}$.

Now let

$$
a=\sum_{t \in G} a_{t} \delta_{t} \in \mathcal{L} .
$$

Then

$$
\begin{aligned}
\|\lambda(a)\| & =\left\|\lambda\left(\sum_{t \in G} a_{t} \delta_{t}\right)\right\| \leq \sum_{t \in G}\left\|\lambda\left(a_{t} \delta_{t}\right)\right\| \leq \sum_{t \in G}\left\|a_{t} \delta_{t}\right\| \\
& =\sum_{t \in G}\left\|a_{t}\right\|=\|a\|_{l_{1}},
\end{aligned}
$$

and so $\|\lambda(a)\| \leq\|a\|_{l_{1}}$, for all $a \in \mathcal{L}$.

We conclude that $\lambda$ is one of the representations over which the sup in the definition of \|\|$a \| \mid$ is taken, and hence $\|\lambda(a)\| \leq\|\| a \| \mid$ for all $a \in \mathcal{L}$.

Remark 2.7. The idea presented above can also be used to show that $l_{1}(\mathrm{G}, \mathrm{A})$ is a core sub-algebra of the full crossed product $A \rtimes_{\alpha} G$ associated to an action of $\mathrm{G}$ on $\mathrm{A}$.

Now that we have established that $\mathcal{L}$ is a core sub-algebra of $\mathrm{C}_{0}(X) \rtimes_{\alpha} G$, all we are left to do, by [6, Proposition 3.4], is to show that $\mathcal{L}$ is isomorphic to $\mathrm{C}_{c}(R)$.

Theorem 2.8. Let $\left(\left\{X_{t}\right\}_{t \in \mathrm{G}},\left\{h_{t \in \mathrm{G}}\right\}\right)$ be a free partial action of a countable group $\mathrm{G}$ over a locally compact Hausdorff space $\mathrm{X}$ such that $X_{t}$ is $\sigma$-compact for every $t,\left(\left\{\mathrm{C}_{0}\left(\mathrm{X}_{t}\right)\right\}_{t \in G},\left\{\alpha_{t}\right\}_{t \in G}\right)$ the corresponding partial action (as defined in the introduction) and $R$ the equivalence relation defined above. Then $\mathcal{L}$ and $\mathrm{C}_{c}(R)$ are isomorphic as ${ }^{*}$-algebras.

Proof. To define a ${ }^{*}$-homomorphism $\rho: \mathcal{L} \rightarrow C_{c}(R)$, we begin defining it as elements of the form $f_{t} \delta_{t}\left(f_{t} \in \mathrm{C}_{0}\left(X_{t}\right)\right)$ and then we 
extend it linearly to $\mathcal{L}$. More precisely, for $f_{t} \delta_{t} \in \mathcal{L}$ and $\left(x, h_{s}(x)\right) \in R$, let

$$
\widetilde{\rho}\left(f_{t} \delta_{t}\right)\left(x, h_{s}(x)\right)= \begin{cases}f_{t}(x) & \text { if } s=t^{-1} \\ 0 & \text { otherwise }\end{cases}
$$

and denote the linear extension of $\widetilde{\rho}$ to $\mathcal{L}$ by $\rho$.

Notice that, since $\mathcal{L}$ consists of finite sums, the set $\{s \in \mathrm{G}$ : $\left.\rho\left(\sum f_{t} \delta_{t}\right)\left(x, h_{s}(x)\right) \neq 0\right\}$ is finite, and hence $\rho$ is well defined (that is, $\rho\left(\sum f_{t} \delta_{t}\right)$ is a continuous function with compact support).

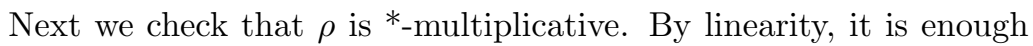
to check this for elements of the form $f_{t} \delta_{t} \in \mathcal{L}$. So, let $f_{t} \delta_{t}, g_{s} \delta_{s} \in \mathcal{L}$ and $\left(x, h_{r}(x)\right) \in R$. Then,

$$
\begin{aligned}
\rho\left(f_{t} \delta_{t} g_{s} \delta_{s}\right)\left(x, h_{r}(x)\right) & =\rho\left(\alpha_{t}\left(\alpha_{t^{-1}}\left(f_{t}\right) g_{s}\right) \delta_{t s}\right)\left(x, h_{r}(x)\right) \\
& = \begin{cases}f_{t}(x) g_{s}\left(h_{t^{-1}}(x)\right) & \text { if } r=(t s)^{-1} \\
0 & \text { otherwise, }\end{cases} \\
& = \begin{cases}f_{t}(x) \rho\left(g_{s} \delta_{s}\right)\left(h_{t^{-1}}(x), h_{r}(x)\right) & \text { if } r=(t s)^{-1} \\
0 & \text { otherwise, }\end{cases} \\
& =\sum_{u: x \in X_{u}-1} \rho\left(f_{t} \delta_{t}\right)\left(x, h_{u}(x)\right) \rho\left(g_{s} \delta_{s}\right)\left(h_{u}(x), h_{r}(x)\right) \\
& =\rho\left(f_{t} \delta_{t}\right) * \rho\left(g_{s} \delta_{s}\right)\left(x, h_{r}(x)\right),
\end{aligned}
$$

and

$$
\begin{aligned}
\rho\left(\left(f_{g} \delta_{g}\right)^{*}\right)\left(x, h_{r}(x)\right) & =\rho\left(\alpha_{g^{-1}}\left(f_{g}^{*}\right) \delta_{g^{-1}}\right)\left(x, h_{r}(x)\right) \\
& = \begin{cases}\alpha_{g^{-1}}\left(f_{g}^{*}\right)(x) & \text { if } r=g \\
0 & \text { otherwise, }\end{cases} \\
& = \begin{cases}\overline{f_{g}\left(h_{r}(x)\right)} & \text { if } r=g \\
0 & \text { otherwise, }\end{cases} \\
& =\frac{\rho\left(f_{g} \delta_{g}\right)\left(h_{r}(x), x\right)}{} \\
& =\left(\rho\left(f_{g} \delta_{g}\right)\right)^{*}\left(x, h_{r}(x)\right) .
\end{aligned}
$$

So $\rho$ is a ${ }^{*}$-homomorphism.

Finally, notice that $\rho$ is also a bijection, since it has an inverse 
$\rho^{-1}: C_{c}(R) \rightarrow \mathcal{L}$ given by $\rho^{-1}(f)=\sum f_{t} \delta_{t}$, where

$$
f_{t}(x)= \begin{cases}f\left(x, h_{t^{-1}}(x)\right) & \text { if }\left(x, h_{t^{-1}}(x)\right) \in R \\ 0 & \text { otherwise. } \square\end{cases}
$$

Remark 2.9. Notice that, since an action of a group on an algebra can be seen as a partial action, the above result is also valid in the context of actions of countable groups over compact spaces.

Corollary 2.10. $\mathrm{C}_{0}(X) \rtimes_{\alpha} G$ is isomorphic to $\mathrm{C}^{*}(R)$.

Proof. Follows from the above theorem and [6, Proposition 3.4].

3. The purely algebraic setting. In this section, we generalize to the purely algebraic setting the results of the previous section and the correspondence between partial dynamical systems and partial $C^{*}$ dynamical systems.

Let $\mathbb{K}$ be a field and $\mathrm{X}$ a set. By an algebra, we mean an associative $\mathbb{K}$-algebra, not necessarily unital. Let $\mathcal{F}_{0}(\mathrm{X})$ denote the algebra of all functions $f: \mathrm{X} \rightarrow \mathbb{K}$ that vanish eventually, that is, $f \in \mathcal{F}_{0}(\mathrm{X})$ if and only if $f(x)=0$ for all but a finite number of $x \in \mathrm{X}$ ( $f$ has finite support), equipped with pointwise operations. Notice that we can see $\mathcal{F}_{0}(\mathrm{X})$ as the direct sum of $\mathbb{K}$ over $\mathrm{X}$, but we will keep the function notation due to its resemblance to the $C^{*}$-setting. Our first goal is to show that there exists a bijective correspondence between $X$ and the set of all non zero homomorphisms from $\mathcal{F}_{0}(\mathrm{X})$ to $\mathbb{K}$, where each $x \in X$ is taken to the homomorphism $\epsilon_{x}: \mathcal{F}_{0}(\mathrm{X}) \rightarrow \mathbb{K}$, given by $\epsilon_{x}(f)=f(x)$, which we call evaluation at $x$.

Proposition 3.1. There exists a bijective correspondence between a set $\mathrm{X}$ and $\widehat{\mathcal{F}_{0}(\mathrm{X})}$ given by:

$$
\begin{aligned}
\mathrm{X} & \longrightarrow \widehat{\mathcal{F}_{0}(\mathrm{X})} \\
x & \longmapsto \epsilon_{x},
\end{aligned}
$$

where $\widehat{\mathcal{F}_{0}(\mathrm{X})}$ denotes the set of all non zero homomorphisms from $\mathcal{F}_{0}(\mathrm{X})$ to $\mathbb{K}$. 
Proof. Given $x \in \mathrm{X}$, let $\delta_{x}$ denote the characteristic function of the set $\{x\}$. Then $\left\{\delta_{x}\right\}_{x \in \mathrm{X}}$ is a $\mathbb{K}$-basis of $\mathcal{F}_{0}(\mathrm{X})$ and $f=\sum_{x \in \mathrm{X}} f(x) \delta_{x}$, for all $f \in \mathcal{F}_{0}(\mathrm{X})$.

First we will prove that there is a bijective correspondence between $\mathbb{K}$-linear maps from $\mathcal{F}_{0}(\mathrm{X})$ to $\mathbb{K}$ and functions from $X$ to $\mathbb{K}$. For this, notice that, if $\phi: \mathcal{F}_{0}(\mathrm{X}) \rightarrow \mathbb{K}$ is a $\mathbb{K}$ linear map and $f \in \mathcal{F}_{0}(\mathrm{X})$, then

$$
\phi(f)=\sum_{x \in \mathrm{X}} f(x) \phi\left(\delta_{x}\right)=\sum_{x \in \mathrm{X}} f(x) \varphi(x)=\sum_{x \in \mathrm{X}} \varphi(x) \epsilon_{x}(f),
$$

where $\varphi: X \rightarrow \mathbb{K}$ is defined by $\varphi(x)=\phi\left(\delta_{x}\right)$, for all $x \in \mathrm{X}$. Conversely, every function $\varphi: \mathrm{X} \rightarrow \mathbb{K}$ defines a $\mathbb{K}$-linear map $\phi: \mathcal{F}_{0}(\mathrm{X}) \rightarrow \mathbb{K}$ via $\phi(f)=\sum_{x \in \mathrm{X}} \varphi(x) \epsilon_{x}(f)$, and it is clear that the correspondence $\phi \leftrightarrow \varphi$ is a bijection.

Now, notice that every evaluation $\epsilon_{x}$ is a nonzero homomorphism. Suppose, conversely, that $\phi: \mathcal{F}_{0}(\mathrm{X}) \rightarrow \mathbb{K}$ is a nonzero homomorphism. Then we have that $\varphi(x) \varphi(y)=\phi\left(\delta_{x}\right) \phi\left(\delta_{y}\right)=\phi\left(\delta_{x} \delta_{y}\right)=\delta_{x, y} \varphi(x)$. Then $\varphi(x) \varphi(y)=0$ if $x \neq y$ and $\varphi(x)^{2}=\varphi(x)$ for all $x \in \mathrm{X}$. Hence, there is exactly one $x \in X$ such that $\varphi(x) \neq 0$, so $\varphi=\epsilon_{x}$.

Our next goal is to show that there is a biunivocal correspondence between partial actions $\left(\left\{X_{t}\right\}_{t \in G}\left\{h_{t}\right\}_{t \in G}\right)$ of a group $G$ in a set $X$ and partial actions $\left(\left\{D_{t}\right\}_{t \in G},\left\{\alpha_{t}\right\}_{t \in G}\right)$ of $G$ in $\mathcal{F}_{0}(\mathrm{X})$. Before we do this, we need a few results.

Proposition 3.2. Let $X$ and $Y$ be sets and $h: X \rightarrow Y$ a bijection. Then the map $\psi_{h}: \mathcal{F}_{0}(Y) \rightarrow \mathcal{F}_{0}(\mathrm{X})$, defined by $\psi_{h}(f)=f \circ h$, is an algebra isomorphism.

Proof. The proof of this proposition is straightforward.

Proposition 3.3. If $\gamma: \mathcal{F}_{0}(Y) \rightarrow \mathcal{F}_{0}(\mathrm{X})$ is an isomorphism, then there exists a unique bijection, $h: X \rightarrow Y$, such that $\gamma=\psi_{h}$, where $\psi_{h}$ is as in the previous proposition.

Proof. Let $\gamma: \mathcal{F}_{0}(Y) \rightarrow \mathcal{F}_{0}(\mathrm{X})$ be an isomorphism. We need to define $h: X \rightarrow Y$. For this, notice that for all $x \in X, \epsilon_{x} \circ \gamma$ is a homomorphism from $\mathcal{F}_{0}(Y)$ to $\mathbb{K}$. By Proposition 3.1, all such 
homomorphisms are evaluations, and hence there exists $y \in Y$ such that $\epsilon_{x} \circ \gamma=\epsilon_{y}$. Define $h(x):=y$.

Notice that indeed $\gamma=\psi_{h}$, since for all $f \in \mathcal{F}_{0}(Y)$ and $x \in X$, we have that $\psi_{h}(f)(x)=f(h(x))=f(y)=\epsilon_{y}(f)=\epsilon_{x} \circ \gamma(f)=$ $\epsilon_{x}(\gamma(f))=\gamma(f)(x)$. Also, $h$ is bijective, since we can define its inverse, $l: Y \rightarrow X$, in the following way: consider $\gamma^{-1}: \mathcal{F}_{0}(\mathrm{X}) \rightarrow \mathcal{F}_{0}(Y)$. Given $y \in Y, \epsilon_{y} \circ \gamma^{-1}$ is a homomorphism from $\mathcal{F}_{0}(\mathrm{X})$ to $\mathbb{K}$ and so there exists $x \in X$ such that $\epsilon_{y} \circ \gamma^{-1}=\epsilon_{x}$. Define $l(y):=x$. Then, for $x \in X$, $l \circ h(x)=l(y)=x_{0}$, where $y$ is such that $\epsilon_{x} \circ \gamma=\epsilon_{y}$ and $x_{0}$ is such that $\epsilon_{y} \circ \gamma^{-1}=\epsilon_{x_{0}}$. So, $x_{0}$ is such that $\epsilon_{x_{0}}=\epsilon_{y} \circ \gamma^{-1}=\epsilon_{x} \circ \gamma \circ \gamma^{-1}=\epsilon_{x}$, and hence $x_{0}=x$ and $l \circ h=I d$. Analogously, we can check tat $h \circ l=I d$, and hence $l=h^{-1}$.

Finally we show that there is a unique $h$ such that $\gamma=\psi_{h}$. For this, suppose that there exist bijections $h_{1}$ and $h_{2}$ such that $\gamma(f)=\psi_{h_{1}}(f)=$ $\psi_{h_{2}}(f)$ for all $f \in \mathcal{F}_{0}(Y)$. We then have that $f \circ h_{1}(x)=f \circ h_{2}(x)$ for all $f \in \mathcal{F}_{0}(Y), x \in X$, which implies that $h_{1}(x)=h_{2}(x)$ for all $x \in X$, since if there exists $x \in X$ such that $h_{1}(x) \neq h_{2}(x)$, then, for $f=\delta_{h_{1}(x)}$, we have that $f\left(h_{1}(x)\right) \neq f\left(h_{2}(x)\right)$.

Proposition 3.4. There is a biunivocal correspondence between non zero ideals of $\mathcal{F}_{0}(\mathrm{X})$ and non empty subsets of $X$.

Proof. Let $I$ be a non zero ideal in $\mathcal{F}_{0}(\mathrm{X})$. Define $A$ as the set of all elements of $X$ such that there exists a function $f \in I$ such that $\delta_{x} \cdot f \neq 0$. Then $I=\mathcal{F}_{0}(A)$, where $\mathcal{F}_{0}(A)$ is included in $\mathcal{F}_{0}(\mathrm{X})$, that is, $\mathcal{F}_{0}(A)=\left\{f \in \mathcal{F}_{0}(\mathrm{X}): f(x)=0\right.$, for all $\left.x \notin A\right\}$.

From the above propositions, we conclude that there is a bijective homomorphism $\psi$ between the set of all bijections from $X$ to $Y$, which we denote by $\operatorname{Bij}(X, Y)$, and the set of all isomorphisms from $\mathcal{F}_{0}(\mathrm{X})$ to $\mathcal{F}_{0}(Y)$, which we denote by Iso $\left(\mathcal{F}_{0}(\mathrm{X}), \mathcal{F}_{0}(Y)\right)$, given by

$$
\begin{aligned}
\psi: \operatorname{Bij}(X, Y) & \longrightarrow \operatorname{Iso}\left(\mathcal{F}_{0}(\mathrm{X}), \mathcal{F}_{0}(Y)\right) \\
h & \longmapsto \psi_{h^{-1}}
\end{aligned}
$$

We are now ready to prove the correspondence between partial actions on a set $X$ and partial actions on $\mathcal{F}_{0}(\mathrm{X})$. 
Proposition 3.5. Let $\theta=\left(\left\{X_{t}\right\}_{t \in G},\left\{h_{t}\right\}_{t \in G}\right)$ be a partial action of a group $G$ in a set $X$, and let $D_{t}=\left\{f \in \mathcal{F}_{0}(\mathrm{X}): f(x)=0\right.$ for all $x \notin$ $\left.X_{t}\right\}$, that is, $D_{t}=\mathcal{F}_{0}\left(X_{t}\right)$. Define $\alpha_{t}: D_{t^{-1}} \rightarrow D_{t}$ by $\alpha_{t}(f)=f \circ h_{t^{-1}}$. Then $\alpha=\left(\left\{D_{t}\right\}_{t \in G},\left\{\alpha_{t}\right\}_{t \in G}\right)$ is a partial action of $G$ in $\mathcal{F}_{0}(\mathrm{X})$, and we say that $\alpha$ arises from $\theta$.

Proof. It is clear that, for each $t \in G, D_{t}$ is an ideal of $\mathcal{F}_{0}(\mathrm{X})$ and, by Proposition 3.2, $\alpha_{t}$ is bijective. We show below that $\left(\left\{D_{t}\right\}_{t \in G},\left\{\alpha_{t}\right\}_{t \in G}\right)$ satisfies the other axioms of the definition of a partial action.

- $D_{e}=\mathcal{F}_{0}(\mathrm{X})$ and $\alpha_{e}=I d$. Since $\theta=\left(\left\{X_{t}\right\}_{t \in G},\left\{h_{t}\right\}_{t \in G}\right)$ is a partial action, we have that $X_{e}=X$ and $h_{e}=I d$ which readily implies that $D_{e}=\mathcal{F}_{0}(\mathrm{X})$ and $\alpha_{e}=I d$.

- $\alpha_{t}\left(D_{t^{-1}} \cap D_{s}\right)=D_{t} \cap D_{t s}$. First, we prove that $\alpha_{t}\left(\mathcal{F}_{0}\left(X_{t^{-1}} \cap\right.\right.$ $\left.\left.X_{s}\right)\right)=\mathcal{F}_{0}\left(h_{t}\left(X_{t^{-1}} \cap X_{s}\right)\right)$. For this, let $y=f \circ h_{t^{-1}}$, for some $f \in \mathcal{F}_{0}\left(X_{t^{-1}} \cap X_{s}\right)$. Now, if $x \notin h_{t}\left(X_{t^{-1}} \cap X_{s}\right)$, then $h_{t^{-1}}(x) \notin X_{t^{-1}} \cap X_{s}$, and hence, $f \circ h_{t^{-1}}(x)=0$ and $y \in \mathcal{F}_{0}\left(h_{t}\left(X_{t^{-1}} \cap X_{s}\right)\right)$. On the other hand, if $f \in \mathcal{F}_{0}\left(h_{t}\left(X_{t^{-1}} \cap\right.\right.$ $\left.\left.X_{s}\right)\right)$, then $f \circ h_{t} \in \mathcal{F}_{0}\left(X_{t^{-1}} \cap X_{s}\right)$ and $\alpha_{t}\left(f \circ h_{t}\right)=f$ as desired.

We can now prove the desired partial action axiom below:

$$
\begin{aligned}
\alpha_{t}\left(D_{t^{-1}} \cap D_{s}\right) & =\alpha_{t}\left(\mathcal{F}_{0}\left(X_{t^{-1}}\right) \cap \mathcal{F}_{0}\left(X_{s}\right)\right) \\
& =\alpha_{t}\left(\mathcal{F}_{0}\left(X_{t^{-1}} \cap X_{s}\right)\right) \\
& =\mathcal{F}_{0}\left(h_{t}\left(X_{t^{-1}} \cap X_{s}\right)\right) \\
& =\mathcal{F}_{0}\left(X_{t} \cap X_{t s}\right) \\
& =\mathcal{F}_{0}\left(X_{t}\right) \cap \mathcal{F}_{0}\left(X_{t s}\right) \\
& =D_{t} \cap D_{t s} .
\end{aligned}
$$

- $\alpha_{t}\left(\alpha_{s}(f)\right)=\alpha_{t s}(f)$ for all $f \in D_{s^{-1}} \cap D_{s^{-1} t^{-1}}$. Let

$$
f \in D_{s^{-1}} \cap D_{s^{-1} t^{-1}}=\mathcal{F}_{0}\left(X_{s^{-1}} \cap \mathrm{X}_{s^{-1} t^{-1}}\right)
$$

and $x \in X_{s^{-1}} \cap \mathrm{X}_{s^{-1}} t^{-1}$. It follows that:

$$
\begin{aligned}
\alpha_{t}\left(\alpha_{s}(f)\right)(x) & =\alpha_{t}\left(f \circ h_{s^{-1}}\right)(x) \\
& =f \circ h_{s^{-1}} \circ h_{t^{-1}}(x) \\
& =f\left(h_{s^{-1}}\left(h_{t^{-1}}(x)\right)\right) \\
& =f\left(h_{s^{-1} t^{-1}}\right)(x) \\
& =\alpha_{t s}(f)(x) .
\end{aligned}
$$


Proposition 3.6. If $\alpha=\left(\left\{D_{t}\right\}_{t \in G},\left\{\alpha_{t}\right\}_{t \in G}\right)$ is a partial action of $G$ in $\mathcal{F}_{0}(\mathrm{X})$, then there exists a partial action $\theta=\left(\left\{X_{t}\right\}_{t \in G},\left\{h_{t}\right\}_{t \in G}\right)$, of $G$ in a set $X$, such that $\alpha$ arises from $\theta$.

Proof. Let $\alpha=\left(\left\{D_{t}\right\}_{t \in G},\left\{\alpha_{t}\right\}_{t \in G}\right)$ be a partial action of $G$ in $\mathcal{F}_{0}(\mathrm{X})$. By Proposition 3.4, we have that each ideal $D_{t}$ is of the form $\mathcal{F}_{0}\left(X_{t}\right)$, for some subset $X_{t}$ of $X$. Now, for each isomorphism $\alpha_{t}: \mathcal{F}_{0}\left(X_{t^{-1}}\right) \rightarrow \mathcal{F}_{0}\left(X_{t}\right)$, we let $h_{t^{-1}}$ be the unique bijection from $X_{t}$ to $X_{t^{-1}}$ such that $\alpha_{t}=\psi_{h_{t-1}}$ (that is, $\alpha_{t}(f)=f \circ h_{t^{-1}}$ as described in Proposition 3.3). This way, we define a partial action $\theta=\left(\left\{X_{t}\right\}_{t \in G},\left\{h_{t}\right\}_{t \in G}\right)$ such that $\alpha$ arises from $\theta$. To finish the proof, we need to we show that $\theta$ is indeed a partial action.

It is straightforward to check that $\theta$ satisfies the first axiom in the definition of a partial action. To verify that $h_{t}\left(X_{t^{-1}} \cap X_{s}\right)=X_{t} \cap X_{t s}$, notice that

$$
\alpha_{t}\left(\mathcal{F}_{0}\left(X_{t^{-1}}\right) \cap \mathcal{F}_{0}\left(X_{s}\right)\right)=\mathcal{F}_{0}\left(X_{t}\right) \cap \mathcal{F}_{0}\left(X_{t s}\right)=\mathcal{F}_{0}\left(X_{t} \cap X_{t s}\right),
$$

and, since the left side on this last equality is equal to $\alpha_{t}\left(\mathcal{F}_{0}\left(X_{t^{-1}} \cap\right.\right.$ $\left.\left.X_{s}\right)\right)=\mathcal{F}_{0}\left(h_{t}\left(X_{t^{-1}} \cap X_{s}\right)\right.$, we obtain the desired equality.

Finally, we prove that $h_{t}\left(h_{s}(x)\right)=h_{t s}(x)$ for all $x \in X_{s^{-} 1} \cap X_{s^{-1} t^{-1}}$. Since $\alpha$ is a partial action, we have that $\alpha_{t}\left(\alpha_{s}(f)\right)=\alpha_{t s}(f)$ for all $f \in \mathcal{F}_{0}\left(X_{s^{-1}} \cap X_{s^{-1} t^{-1}}\right)$. But this implies that $\psi_{h_{t s}}(f)=\psi_{h_{t} \circ h_{s}}(f)$ for all $f \in \mathcal{F}_{0}\left(X_{s^{-1}} \cap X_{s^{-1} t^{-1}}\right)$, and hence $h_{t s}=h_{t} \circ h_{s}$ for all $x \in X_{s^{-1}} \cap X_{s^{-1} t^{-1}}$, as desired.

We now focus on realizing the partial skew group $\operatorname{ring} \mathcal{F}_{0}(\mathrm{X}) \rtimes_{\alpha} G$, associated to a partial action $\alpha$ of a group $G$ in $\mathcal{F}_{0}(\mathrm{X})$, as an algebra from an equivalence relation.

For the reader's convenience, we recall the definition of a partial skew group ring, as defined in [3], below.

Definition 3.7. Let $\alpha$ be a partial action of the group $G$ in the algebra $A$. The partial skew group ring, $A \rtimes_{\alpha} G$ associated to $\alpha$ is defined as the set of all finite formal sums $\sum_{t \in G} a_{t} \delta_{t}$ where, for all $t \in G, a_{t} \in D_{t}$ and $\delta_{t}$ are symbols. Addition is defined in the usual way and multiplication is determined by

$$
\left(a_{t} \delta_{t}\right)\left(b_{s} \delta_{s}\right)=\alpha_{t}\left(\alpha_{t^{-1}}\left(a_{t}\right) b_{s}\right) \delta_{t s}
$$


Recall that, for every partial action in $\mathcal{F}_{0}(\mathrm{X})$, there is a partial action associated in $X$. The set level is what we need to define the corresponding equivalence relation. So, let $\left(\left\{X_{t}\right\}_{t \in G},\left\{h_{t}\right\}_{t \in G}\right)$ be a free partial action of $G$ in $X$. We define the equivalence relation $R$ in $X$ as

$$
R=\left\{\left(x, h_{t}(x)\right) \in X \times X: x \in X_{t^{-1}}, t \in G\right\},
$$

and equip $\mathcal{F}_{0}(R)=\{f: R \rightarrow \mathbb{K}: f$ eventually vanishes $\}$ with operations defined by:

$$
\begin{aligned}
(k f+g)\left(x, h_{t}(x)\right) & =k f\left(x, h_{t}(x)\right)+g\left(x, h_{t}(x)\right), \\
(f * g)\left(x, h_{t}(x)\right) & =\sum_{s \in G} f\left(x, h_{s}(x)\right) g\left(h_{s}(x), h_{t}(x)\right),
\end{aligned}
$$

for all $f, g \in \mathcal{F}_{0}(R), k \in \mathbb{K}$ and $\left(x, h_{t}(x)\right) \in R$.

Theorem 3.8. If $\theta=\left(\left\{X_{t}\right\}_{t \in G},\left\{h_{t}\right\}_{t \in G}\right)$ is a free partial action of a group $G$ in a set $X$, and $\alpha=\left(\left\{D_{t}\right\}_{t \in G},\left\{\alpha_{t}\right\}_{t \in G}\right)$ is the associated partial action of $G$ in $\mathcal{F}_{0}(\mathrm{X})$, then the algebras $\mathcal{F}_{0}(R)$ and $\mathcal{F}_{0}(\mathrm{X}) \rtimes_{\alpha} G$ are isomorphic.

Proof. To prove the theorem, we will define an isomorphism $\Gamma$ from $\mathcal{F}_{0}(\mathrm{X}) \rtimes_{\alpha} G$ to $\mathcal{F}_{0}(R)$. For elements of the form $f \delta_{t} \in \mathcal{F}_{0}(\mathrm{X}) \rtimes_{\alpha} G$, we let $\Gamma\left(f \delta_{t}\right)=\tilde{f}$, where

$$
\widetilde{f}(x, y)= \begin{cases}f(x) & \text { if } y=h_{t^{-1}}(x) \\ 0 & \text { otherwise, }\end{cases}
$$

and we define $\Gamma$ in $\mathcal{F}_{0}(\mathrm{X}) \rtimes_{\alpha} G$ by extending it linearly.

Notice that $\Gamma$ is well defined since, if $\left\{x_{1}^{t}, \cdots, x_{n_{t}}^{t}\right\}$ is the finite support of $f_{t} \in \mathcal{F}_{0}(\mathrm{X})$, then $\Gamma\left(f_{t} \delta_{t}\right)$ has finite support given by the set $\left\{\left(x_{1}^{t}, h_{t^{-1}}\left(x_{1}^{t}\right)\right), \ldots,\left(x_{n_{t}}^{t}, h_{t^{-1}}\left(x_{n_{t}}^{t}\right)\right)\right\}$.

Now we show that $\Gamma$ is an isomorphism of $\mathbb{K}$-algebras. For this, it is enough to show that $\Gamma$ is bijective and preserves the product, that is,

$$
\begin{aligned}
& \Gamma\left(f_{t} \delta_{t} f_{g} \delta_{g}\right)=\Gamma\left(f_{t} \delta_{t}\right) * \Gamma\left(f_{g} \delta_{g}\right), \\
& \text { for all } f_{t} \delta_{t}, f_{g} \delta_{g} \in \mathcal{F}_{0}(\mathrm{X}) \rtimes_{\alpha} G .
\end{aligned}
$$

Notice that

$$
\Gamma\left(f_{t} \delta_{t} f_{s} \delta_{s}\right)(x, y)=\Gamma\left(\alpha_{t}\left(\alpha_{t^{-1}}\left(f_{t}\right) f_{s}\right) \delta_{t s}\right)(x, y)
$$




$$
\begin{aligned}
& = \begin{cases}\alpha_{t}\left(\alpha_{t^{-1}}\left(f_{t}\right) f_{s}\right)(x), & \text { if } y=h_{(t s)^{-1}}(x), \\
0 & \text { otherwise, }\end{cases} \\
& = \begin{cases}f_{t}(x)\left(f_{s}\right)\left(h_{t^{-1}}(x)\right), & \text { if } y=h_{(t s)^{-1}}(x), \\
0 & \text { otherwise, }\end{cases}
\end{aligned}
$$

and, on the other hand,

$$
\begin{aligned}
\Gamma\left(f_{t} \delta_{t}\right) * \Gamma\left(f_{s} \delta_{s}\right)(x, y) & =\sum_{r \in G} \Gamma\left(f_{t} \delta_{t}\right)\left(x, h_{r}(x)\right) * \Gamma\left(f_{s} \delta_{s}\right)\left(h_{r}(x), y\right), \\
& =f_{t}(x) \Gamma\left(f_{s} \delta_{s}\right)\left(h_{t^{-1}}(x), y\right), \\
& = \begin{cases}f_{t}(x)\left(f_{s}\right)\left(h_{t^{-1}}(x)\right), & \text { if } y=h_{s^{-1}}\left(h_{t^{-1}}(x)\right), \\
0 & \text { otherwise, }\end{cases} \\
& = \begin{cases}f_{t}(x)\left(f_{s}\right)\left(h_{t^{-1}}(x)\right), & \text { if } y=h_{(t s)^{-1}}(x), \\
0 & \text { otherwise, }\end{cases}
\end{aligned}
$$

We conclude that $\Gamma\left(f_{t} \delta_{t} f_{s} \delta_{s}\right)=\Gamma\left(f_{t} \delta_{t}\right) * \Gamma\left(f_{s} \delta_{s}\right)$.

Next, we prove injectivity. For this, suppose that $\Gamma\left(\sum_{t \in G} f_{t} \delta_{t}\right)=0$. Notice that, since $\theta$ is a free, if $\Gamma\left(f_{t} \delta_{t}\right)(x, y) \neq 0$, then $\Gamma\left(f_{s} \delta_{s}\right)(x, y)=0$ for all $s \neq t$. So,

$$
\sum_{t \in G} \Gamma\left(f_{t} \delta_{t}\right)\left(x, h_{s}(x)\right)=0, \quad \text { for all } x \in X_{s^{-1}}, s \in G,
$$

implies that $f_{s^{-1}}(x)=0$ for all $x \in X_{s^{-1}}, s \in G$. We infer that

$$
f_{t} \equiv 0 \quad \text { for all } t \in G
$$

and hence,

$$
\sum_{t \in G} f_{t} \delta_{t}=0
$$

as desired.

Finally, we prove that $\Gamma$ is surjective. For $f \in \mathcal{F}_{0}(R)$, we define $f_{t}(x):=f\left(x, h_{t^{-1}}(x)\right)$, for all $x \in X_{t}$. Notice that

$$
\sum_{i=1}^{n} f_{t_{i}} \delta_{t_{i}} \in \mathcal{F}_{0}(\mathrm{X}) \rtimes_{\alpha} G
$$


and

$$
\Gamma\left(\sum_{i=1}^{n} f_{t_{i}} \delta_{t_{i}}\right)=f
$$

so that $\Gamma$ is surjective.

By the above exposition, we conclude that

$$
\mathcal{F}_{0}(\mathrm{X}) \rtimes_{\alpha} G \text { and } \mathcal{F}_{0}(R)
$$

are isomorphic as $\mathbb{K}$-algebras.

Remark 3.9. Partial skew group rings similar to the ones considered in the above theorem have appeared before, see [12].

One important issue that has to be handled when dealing with partial skew group rings concerns the associativity question, as partial skew group rings may or may not be associative, see [3]. In the case of $\mathcal{F}_{0}(\mathrm{X}) \rtimes_{\alpha} G$ the answer is affirmative, that is, $\mathcal{F}_{0}(\mathrm{X}) \rtimes_{\alpha} G$ is always associative, since all the ideals of $\mathcal{F}_{0}(\mathrm{X})$ are idempotent (see $[\mathbf{3}, \mathbf{1 9}]$ for associativity criteria). Below, we give a quick proof that $\mathcal{F}_{0}(\mathrm{X}) \rtimes_{\alpha} G$ is always associative, one that does not depend on the theory developed in $[3,19]$.

Proposition 3.10. $\mathcal{F}_{0}(R)$ is an associative $\mathbb{K}$-algebra.

Proof. Let $f, g, l \in \mathcal{F}_{0}(R)$ and $\left(x, h_{t}(x)\right) \in R$. Then:

$$
\begin{aligned}
& (f * g) * l\left(x, h_{t}(x)\right) \\
& =\sum_{s \in G}(f * g)\left(x, h_{s}(x)\right) l\left(h_{s}(x), h_{t}(x)\right) \\
& =\sum_{s \in G}\left(\sum_{r \in G} f\left(x, h_{r}(x)\right) g\left(h_{r}(x), h_{s}(x)\right)\right) l\left(h_{s}(x), h_{t}(x)\right) \\
& =\sum_{s \in G} \sum_{r \in G}\left[f\left(x, h_{r}(x)\right) g\left(h_{r}(x), h_{s}(x)\right)\right] l\left(h_{s}(x), h_{t}(x)\right) \\
& =\sum_{s \in G} \sum_{r \in G} f\left(x, h_{r}(x)\right)\left[g\left(h_{r}(x), h_{s}(x)\right) l\left(h_{s}(x), h_{t}(x)\right)\right] \\
& \text { since } \mathbb{K} \text { is associative }
\end{aligned}
$$




$$
\begin{aligned}
& =\sum_{r \in G} f\left(x, h_{r}(x)\right) \sum_{s \in G} g\left(h_{r}(x), h_{s}(x)\right) l\left(h_{s}(x), h_{t}(x)\right) \\
& =\sum_{r \in G} f\left(x, h_{r}(x)\right) \sum_{s \in G} g * l\left(h_{r}(x), h_{t}(x)\right) \\
& =f *(g * l)\left(x, h_{t}(x)\right) .
\end{aligned}
$$

So

$$
(f * g) * l=f *(g * l),
$$

and $\mathcal{F}_{0}(R)$ is associative.

Corollary 3.11. $\mathcal{F}_{0}(\mathrm{X}) \rtimes_{\alpha} G$ is an associative $\mathbb{K}$-algebra.

To finalize the paper, we use Theorem 3.8 to characterize the ideals in $\mathcal{F}_{0}(\mathrm{X}) \rtimes_{\alpha} G$ in terms of $R$-invariant sets:

Definition 3.12. A subset $Z \subseteq X$ is said to be $R$-invariant if, whenever $(z, x) \in R$, with $z \in Z$, then $x \in Z$.

Proposition 3.13. Let $I$ be an ideal in $\mathcal{F}_{0}(R)$. Then there exists an $R$-invariant set $Z$ such that $I=\mathcal{F}_{0}((Z \times Z) \cap R)$.

Proof. First notice that, if $f$ is a non zero function in $I$ and $f\left(y, h_{t}(y)\right) \neq 0$, then $\delta_{\left(y, h_{t}(y)\right)} \in I$. This follows because

$$
\delta_{\left(y, h_{t}(y)\right)}=\delta_{(y, y)} * f * \delta_{\left(h_{t}(y), h_{t}(y)\right)} .
$$

Also notice that

$$
\delta_{\left(y, h_{t}(y)\right)} * \delta_{\left(z, h_{s}(z)\right)}=0
$$

if $z \neq h_{t}(y)$ and

$$
\delta_{\left(y, h_{t}(y)\right)} * \delta_{\left(z, h_{s}(z)\right)}=\delta_{\left(y, h_{s t}(y)\right)}
$$

if $z=h_{t}(y)$.

With the above in mind, let $I$ be an ideal in $\mathcal{F}_{0}(R)$. Define

$Z=\left\{z \in X:\right.$ there exists $f \in I$ and $t \in G$ such that $\left.f\left(z, h_{t}(z)\right) \neq 0\right\}$.

We will prove first that $Z$ is $R$-invariant. For this, let $z \in Z$ and $\left(z, h_{s}(z)\right) \in R$. Then, by the definition of $Z$, there exists $f \in I$ and 
$t \in G$ such that $f\left(z, h_{t}(z)\right) \neq 0$ and, by the first paragraph in this proof, we have that $\delta_{\left(z, h_{t}(z)\right)} \in I$. But then

$$
\delta_{\left(z, h_{t}(z)\right)} * \delta_{\left(h_{t}(z), h_{s t^{-1}}(z)\right)}=\delta_{\left(z, h_{s}(z)\right)} \in I .
$$

We conclude that

$$
\delta_{\left(h_{s}(z), z\right)} * \delta_{\left(z, h_{s}(z)\right)}=\delta_{\left(h_{s}(z), h_{s}(z)\right)} \in I,
$$

and hence, $h_{s}(z) \in Z$, as desired.

Next, we prove that $\mathcal{F}_{0}((Z \times Z) \cap R) \subseteq I$. Notice that, for this, it is enough to show that for each $\left(x, h_{t}(x)\right) \in(Z \times Z) \cap R$ the associated delta Dirac function $\delta_{\left(x, h_{t}(x)\right)}$ belongs to $I$. So, let $\left(x, h_{t}(x)\right) \in(Z \times Z) \cap R$. Then, since $x \in Z$, there exists $f \in I$ and $s \in G$ such that $f\left(x, h_{s}(x)\right) \neq 0$, and hence, $\delta_{\left(x, h_{s}(x)\right)} \in I$. We conclude that

$$
\delta_{\left(x, h_{t}(x)\right)}=\delta_{\left(x, h_{s}(x)\right)} * \delta_{\left(h_{s}(x), h_{t s^{-1}}(x)\right)}
$$

belongs to $I$ as desired.

Finally, $I \subseteq \mathcal{F}_{0}((Z \times Z) \cap R)$ since, if $f \in I$ and $f\left(\left(x, h_{t}(x)\right)\right) \neq 0$, then $z \in Z$, and hence, by the $R$-invariance of $Z, h_{t}(z) \in Z$. So the support of $f$ is contained in $Z \times Z$.

Remark 3.14. Notice that, if $Z$ is an $R$-invariant subset, then $\mathcal{F}_{0}((Z \times$ $Z) \cap R$ ) is an ideal of $\mathcal{F}_{0}(R)$.

Remark 3.15. Recently, Leavitt path algebras of countable graphs were characterized as partial skew group rings, see [12]. In the case of finite graphs with no cycles, the partial skew group ring introduced in [12] arises from a free partial action on a set. So, for these graphs, one can apply the last result above to obtain the characterization of the ideals of the associated Leavitt path algebras given by Tomforde in [20]. We refrain from providing the details as this would require the introduction of many notions not mentioned here.

\section{ENDNOTES}

1. By a representation of a *algebra $B$ we mean a multiplicative, *-preserving, linear map $\pi: B \rightarrow \mathcal{B}(H)$, where $H$ is a Hilbert space. 


\section{REFERENCES}

1. F. Abadie, On partial actions and groupoids, Proc. Amer. Math. Soc. 132 (2004), 1037-1047.

2. J. Ávila and M. Ferrero, Closed and prime ideals in partial skew group rings of abelian groups, J. Alg. Appl. 10 (2011), 96-1-978.

3. M. Dokuchaev and R. Exel, Associativity of crossed products by partial actions, enveloping actions and partial representations, Trans. Amer. Math. Soc. 357 (2005), 1931-1952.

4. R. Exel, Circle actions on $C^{*}$-algebras, partial automorphisms and a generalized Pimsner-Voiculescu exact sequence, J. Funct. Anal. 122 (1994), 361-401.

5. Partial actions of groups and actions of semigroups, Proc. Amer. Math. Soc. 126 (1998), 3481-3494.

6. R. Exel, T. Giordano and D. Gonçalves, Enveloping algebras of partial actions as groupoid $C^{*}$-algebras, J. Oper. Th. 65 (2011), 197-210.

7. M. Ferrero and J. Lazzarin, Partial actions and partial skew group rings, J. Alg. 319 (2008), 5247-5264.

8. D. Gonçalves, New $C^{*}$-algebras from substitution tilings, J. Oper. Th. 57 (2007), 391-407.

9. J. Funct. Anal. 260 (2011), 998-1019.

10. Simplicity of partial skew group rings of abelian groups, Canad. Math. Bull. 57 (2014), 511-519.

11. D. Gonçalves and D. Royer, On the representations of Leavitt path algebras, J. Alg. 333 (2011), 258-272.

12. L Leavitt path algebras as partial skew group rings, Comm. Alg. 42 (2014), 3578-3592.

13. D. Gonçalves, J. Öinert and D. Royer, Simplicity of partial skew group rings with applications to Leavitt path algebras and topological dynamics, J. Alg. 420 (2014), 201-216.

14. K. McClanahan, K-theory for partial crossed products by discrete groups, J. Funct. Anal. 130 (1995), 77-117.

15. J. Öinert, Simple group graded rings and maximal commutativity, Contemp. Math. 503, American Mathematical Society, Providence, RI, 2009.

16. Simplicity of skew group rings of abelian groups, Comm. Alg. 42 (2014), 831-841.

17. I.F. Putnam, A survey of recent $K$-theoretic invariants for dynamical systems, in The dynamics of $Z^{d}$-actions, M. Pollicott and K. Schmidt, eds., Cambridge University Press, Cambridge, 1996.

18. Jean N. Renault, A groupoid approach to $C^{*}$-algebras, Lect. Notes Math. 793, Springer-Verlag, New York, 1980.

19. B.T. Shourijeh and M.A. Faraji, Strong associativity of a group algebra, Expo. Math. 24 (2006), 379-383. 
20. M. Tomforde, Uniqueness theorems and ideal structure for Leavitt path algebras, J. Alg. 318 (2007), 270-299.

Department of Mathematics, Universidade do Estado de Santa Catarina, JoINVILle, 89219-710, BRAZIL

Email address: vivibeuter@gmail.com

Department of Mathematics, Universidade Federal de Santa Catarina, Florianópolis, 88040-900, BraziL

Email address: daemig@gmail.com 